Rev. High Pressure Sci. Technol., Vol. 7 (1998) 55 58

\title{
Structures of FeS Polymorphs at High Pressure and Temperature
}

\author{
Y. Fei ${ }^{*}$, C. T. Prewitt ${ }^{*}$, D. J. Frost ${ }^{*}$, J. B. Parise ${ }^{\dagger}$, K. Brister ${ }^{\dagger \dagger}$ \\ ${ }^{*}$ Geophysical Laboratory and Center for High Pressure Research, \\ Carnegie Institution of Washington, Washington, DC 20015, USA \\ †Earth and Space Sciences, SUNY, Stony Brook, NY 11794, USA \\ ${ }^{\dagger}$ CHESS, Cornell University, Ithaca, NY 14853, USA
}

\begin{abstract}
Powder $\mathrm{x}$-ray diffraction data for FeS polymorphs were collected at high pressure and temperature, using monochromatic synchrotron $\mathrm{x}$-radiation and image plate. The diffraction data collected at $10.95 \mathrm{GPa}$ and $300 \mathrm{~K}$ revealed a monoclinic high-pressure phase (FeS III) with cell parameters, $a=8.025(4) \AA, b=5.614(2) \AA, c=6.414(4) \AA$, and $\beta=93.01(4)^{\circ}$. The space group of this monoclinic phase is $P 2_{1} / a$. We also collected diffraction data for the high $P T$ phase (FeS IV) at 5.2 GPa and $9.0 \mathrm{GPa}$ and at $530 \mathrm{~K}$ and for the high- $T$ phase (FeS V) at $3.4 \mathrm{GPa}$ and $650 \mathrm{~K}$. The FeS IV phase has a hexagonal $(2 a, c)$ unit cell (space group $P 6_{3} m c$ ) and the FeS V phase has a simple NiAs-type structure (space group $P 6_{3} / \mathrm{mmc}$ ). The structure parameters were determined by Rietveld refinements.

[crystal chemistry, phase transition, crystallography, synchrotron x-ray diffraction, high-pressure physics]
\end{abstract}

\section{Introduction}

On the basis of cosmochemical and petrologic arguments, it has been proposed that sulfur may be the lighter alloying element in the cores of the Earth and other terrestrial planets such as Mars. Knowledge of the various forms, or polymorphs, of iron sulfide (FeS) that occur at high pressure and high temperature, and their physical properties, is of particular importance in understanding the composition and temperature of the planetary cores. The phase diagram of FeS at high pressure and temperature has been determined using energydispersive $x$-ray diffraction data $[1,2,3]$. Five $\mathrm{FeS}$ polymorphs were identified. The diffraction patterns of the high- $P$ (FeS III), high-PT (FeS IV), and high- $T$ (FeS V) phases can be indexed on a monoclinic, a $(2 a, c)$ hexagonal, and a simple hexagonal unit cell, respectively. Whereas the $d$-spacings derived from diffraction data determine the unit cell dimension, the intensity data provide information on atomic arrangement of a crystal. Because the diffraction data obtained by the energy-dispersive technique do not provide accurate intensity information, the atomic arrangements of the high-pressure polymorphs, FeS III, FeS IV, and FeS V, are still unknown. In order to solve the crystal structures of FeS polymorphs at high $P$ and $T$, we have conducted in situ synchrotron $\mathrm{x}$-ray diffraction measurements on FeS using the image plate technique. In this paper, we will compare the diffraction data obtained by energy-dispersive and image plate techniques and report structure parameters of the high-pressure polymorphs obtained by Rietveld refinements.

\section{Experimental Procedure}

The experiments were carried out in an externally heated diamond-anvil cell that is capable of achieving pressures greater than $125 \mathrm{GPa}$ at temperatures up to $1100 \mathrm{~K}$ in a mildly reducing atmosphere (Ar with $1 \% \mathrm{H}_{2}$ ) $[2,4,5]$. A pair of 600 $\mu \mathrm{m}$ flat diamond anvils was used in this experiment. The FeS powder sample was loaded in a sample chamber drilled from a preindented rhenium gasket. The initial sample chamber dimension was $250 \mu \mathrm{m}$ in diameter by $103 \mu \mathrm{m}$ in thickness. Less than one-third of the chamber volume was filled with FeS powder sample. The rest of the volume was filled with neon gas at about $200 \mathrm{MPa}$ in a high-pressure gas-loading device. The pressure gradient across the sample chamber was minimized under the neon pressure medium environment. Small ruby grains and gold foil were placed in the sample chamber as pressure calibrants. The sample was heated with a large sleeve-shaped platinum-wire heater fitted around the protruding portion of the piston-cylinder and a small molybdenum-wire heater positioned around the diamond anvils. Temperatures were measured with a Pt/Pt-10\%Rh thermocouple placed near the sample chamber.

The sample was initially compressed to $10.95 \mathrm{GPa}$ at room temperature. In situ $\mathrm{x}$-ray diffraction measurements were made during the room-temperature compression at the X17C beamline, National Synchrotron Light Source, Brookhaven National Laboratory. The observed phase transformations in FeS at room temperature were consistent with those reported in our previous papers $[1,2]$. In order to improve the resolution and obtain accurate intensity information, we conducted in situ $\mathrm{x}$-ray diffraction measurements on the same sample, using monochromatic synchrotron $x$-radiation and image plate at the Cornell High Energy Synchrotron Source (CHESS). The diffraction data were recorded on a Fuji image plate and read with a Fuji scanner. The details of the image plate technique have been described elsewhere [6]. The experimental setup at CHESS was similar to that of Kunz et al. [7], except that we used a high-temperature cell with a $90^{\circ}$ open slot. A monochromatic beam of $20 \mathrm{keV}(\lambda=0.6199 \AA)$ was used in the experiments. The sample-plate distance was $309.3 \mathrm{~mm}$, determined by measuring the lattice parameter of gold at a known pressure. With this setup, the recorded image dimension is $256 \mathrm{~mm}$ by $50 \mathrm{~mm}$ with $100 \mu \mathrm{m}$ resolution. The image data were reduced to an intensity vs. $2 \theta$ (or $d$-spacing) plot with an IMP program (imaging plate analysis package developed by $\mathrm{K}$. Brister). The cut-off $d$-spacing is about $1.60 \AA$.

\section{Results and Discussion}

Monoclinic high-pressure phase, FeS III

A high-pressure $\mathrm{FeS}$ phase (FeS III) forms at pressures above $6.7 \mathrm{GPa}$ at room temperature [8, 9]. The stability field of 
this phase was summarized by Fei et al. [1]. The structure assignment of this phase has been controversial. Pichulo [10] and Mao et al. [11] initially assigned an orthorhombic unit cell to this high-pressure phase, although the fit to their $\mathrm{x}$-ray diffraction data was not perfect. Prewilt and Fei (see Reference and Notes in (1]) proposed a monoclinic unit cell on which several diffraction lines could not be indexed. Recently, Kusaba $\mathrm{cl}$ al. [3] indexed their energy-dispersive $x$-ray diffraction data, collected at $10.30 \mathrm{GPa}$ and $300 \mathrm{~K}$, based on a monoclinic unit cell with cell parameters, $a=8.044(3) \AA, b=5.611(2) \AA, c=$ $6.433(4) \AA$, and $\beta=93.11(4)^{\circ}$. We obtained $x$-ray diffraction data for the high-pressure phase FeS III at $10.95 \mathrm{GPa}$ and 300 $\mathrm{K}$ by using both energy-dispersive and image plate techniques. Figure 1 compares the diffraction patterns collected at the same $P-T$ condition by both techniques. The spectrum derived from the image plate data shows much higher resolution than the energy-dispersive spectra. The image plate data also contain important intensity information, whereas the intensities in the energy-dispersive spectra vary depending on a number of factors. We indexed the image plate diffraction data on a monoclinic unit cell and obtained cell parameters, $a=8.025(4)$ $\AA, b=5.614(2) \AA, c=6.414(4) \AA$, and $\beta=93.01(4)^{\circ}$, similar to the values determined by Kusaba et al. [3].

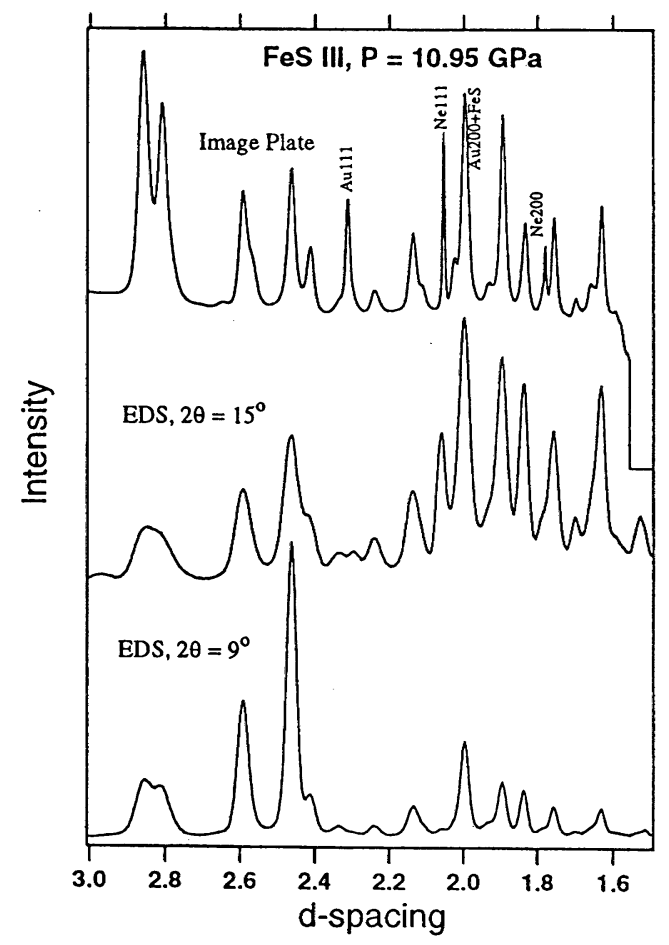

Fig. 1. Comparison of diffraction spectra obtained by energydispersive and image plate techniques. $d$-values larger than 3.0 $\AA$ were not shown.
The intensity data for the high-pressure FeS III phase obtained in this study can be used to test some of the proposed structure models. Kusaba et al [3] proposed $P 2_{1}$ or $P 2_{1} / m$ as possible space groups for the monoclinic phase FeS III. The calculated intensities based on those structure models cannot match the measured intensities. However, the observed $x$-ray diffraction pattern can be interpreted by a structure model with a space group $P 2_{1} / a$ proposed by Nelincs et al [12]. The refined unit cell parameters for FeS III are $a=8.025(4) \AA, b=5.614(2)$ $\AA, c=6.414(4) \AA$, and $\beta=93.01(4)^{\circ}$ (Figure 2).

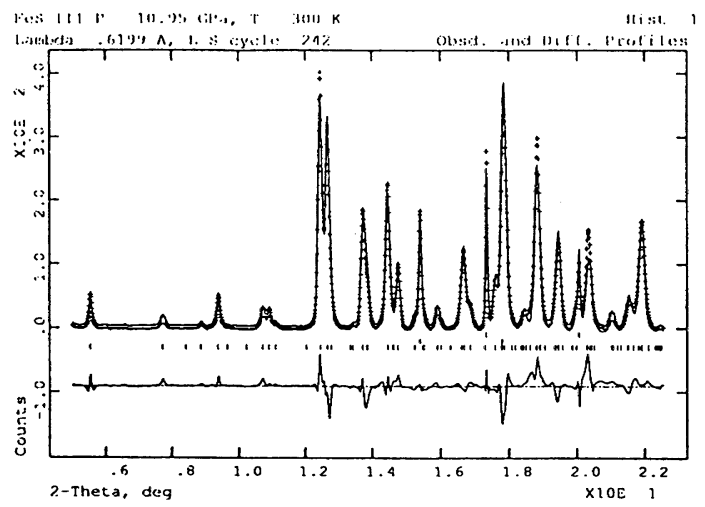

Fig. 2. Observed (crosses) and calculated (solid line) x-ray diffraction pattem for the monoclinic high-pressure phase of $\mathrm{FeS}$ at $10.95 \mathrm{GPa}$ and $300 \mathrm{~K}$. Tick marks for gold (pressure calibrant), neon (pressure medium), and the FeS phase are shown below the pattern. The difference curve is shown at the bottom. The refinement was based on space group $P Z_{1} / a$ with cell parameters, $a=8.025(4) \AA, b=5.614(2) \AA, c=6.414(4)$ $\AA$, and $\beta=93.01(4)^{\circ}$.

\section{Hexagonal high-pressure-temperature phase, $\mathrm{FeS} I \mathrm{~V}$}

Upon heating, the monoclinic phase FeS III transforms to a NiAs-type hexagonal structure with a $(2 a, c)$ unit cell (assigned as FeS IV) [1, 2]. The high-PT FeS IV phase is stable over a wide $P T$ range. However, within the stability field of the $\mathrm{FeS}$ IV, an abrupt shortening of the $c$ axis at about $6 \mathrm{GPa}$ was observed [1]. This abrupt shortening of the $c$ axis was not accompanied by any structure change, based on the energydespersive diffraction data, but it result in a more efficiently packed hexagonal structure. Fei et al. [1] attributed this change to an electronic transition.

In order to understand the nature of this change, we need to determine the structure details of the high- $P T$ FeS IV phase at pressures below and above $6 \mathrm{GPa}$. we collected $\mathrm{x}$-ray diffraction data for FeS IV at 5.4 and $9.0 \mathrm{GPa}$ and at a temperature of 530 $\mathrm{K}$, using the image plate technique. The diffraction data agree with our previous result [1] that FeS IV has a hexagonal unit cell with a $(2 a, c)$ unit cell. The determined cell parameters are $2 a=6.7175 \AA$ and $c=5.6542 \AA$ at $5.2 \mathrm{GPa}$ and $2 a=6.5879 \AA$ 
and $c=5.3998 \AA$ at $9.0 \mathrm{GPa}$. The new data confirmed the abrupt shortening of the $c$ axis at about $6 \mathrm{GPa}$ (Figure 3), observed in our previous study [1,2].

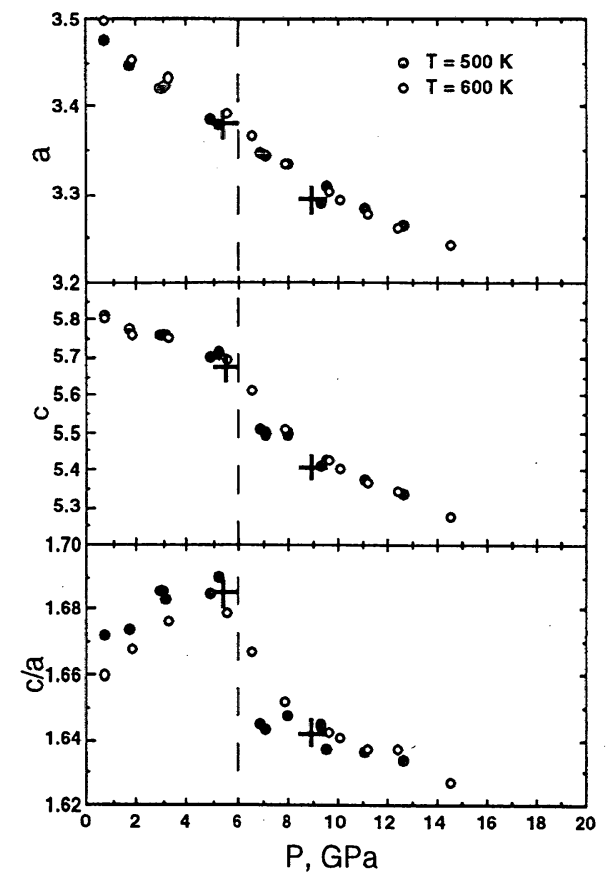

Fig. 3. NiAs-type substructure lattice parameters $a$ and $c$ and the $c / a$ ratio of FeS IV $(2 a, c)$ as a function of pressure. Data at $500 \mathrm{~K}$ (solid circles) and $600 \mathrm{~K}$ (open circles) were collected using an energy-dispersive $\mathrm{x}$-ray diffraction technique $[1,2]$ and data at $530 \mathrm{~K}$ (crosses) were collected using an image plate (this study).

The $x$-ray diffraction patterns of the FeS IV phase, collected at high pressure and temperature, were used for structure refinement. We used a structure model with a space group $\mathrm{P}_{3} \mathrm{mc}$, the same model used for a high-temperature $\mathrm{FeS}$ phase by Keller-Besrest and Collin [13]. The Rietveld refinement, using the program package GSAS [14], yielded reasonably good agreement between the observed and calculated $x$-ray diffraction pattern (Figs. 4a, b). The refined parameters are listed in Tables 1a and $1 \mathrm{~b}$. Both refinements showed similar intensity misfit, especially for reflections 200 and 220. Refinements including a preferred orientation model would improve the fit.

The structure refinement confirmed out previous conclusion that the crystal structure of FeS IV is the same at pressures below and above $6 \mathrm{GPa}$, despite the contraction of the $c$-axis across $6 \mathrm{GPa}$. The $4 \%$ density change, resulted from the contraction of the $c$-axis, may be explained by an electronic/magnetic transition.
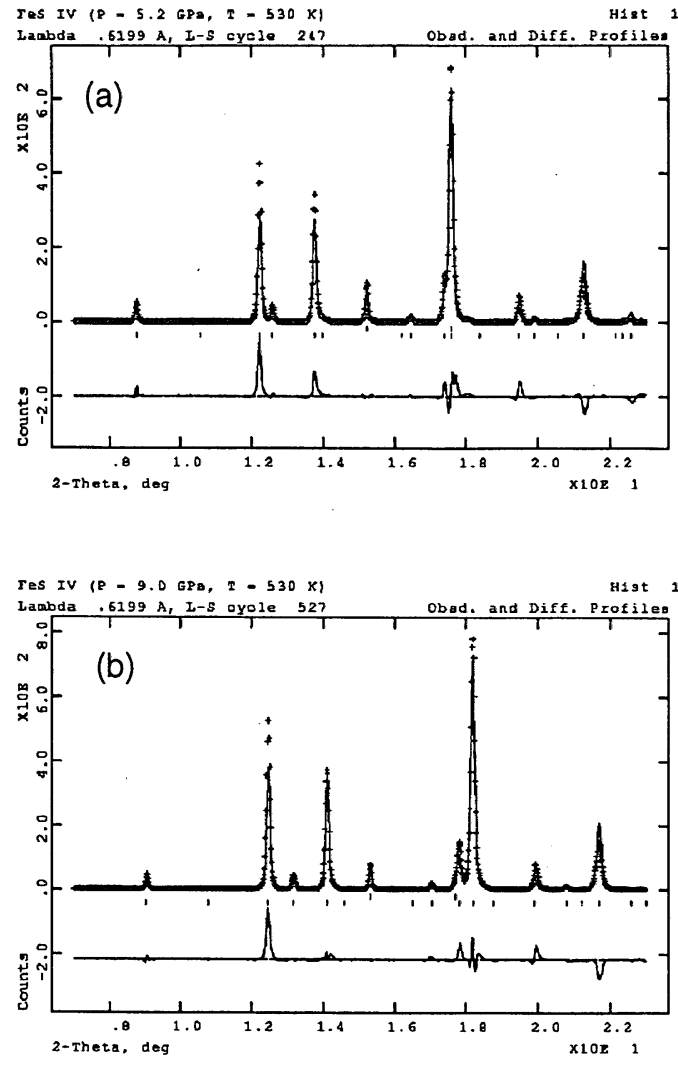

Fig. 4. Observed (crosses) and calculated (solid line) x-ray diffraction pattern for FeS IV (a) at $5.2 \mathrm{GPa}$ and $530 \mathrm{~K}$, and (b) at $9.0 \mathrm{GPa}$ and $530 \mathrm{~K}$. Tick marks for gold (pressure calibrant) and the FeS IV phase are shown below the pattern. The difference curve is shown at the bottom.

Table 1a. Refined parameters for FeS IV phase at $5.2 \mathrm{GPa}$ and $530 \mathrm{~K}$ derived from Rietveld refinement.

\begin{tabular}{lllll} 
atom & $\mathrm{x}$ & $\mathrm{y}$ & $\mathrm{z}$ & $U_{\text {iso }} \mathrm{x} 10^{2}$ \\
\hline
\end{tabular}

$\begin{array}{lllll}\mathrm{Fe}(1) & 0.5208 & 0.0416 & 0 & 2.2 \\ \mathrm{Fe}(2) & 0 & 0 & -0.0227 & 3.5 \\ \mathrm{~S}(1) & 0.1602 & 0.3204 & 0.2295 & 1.7 \\ \mathrm{~S}(2) & 0.3333 & 0.6667 & 0.7747 & 0.8\end{array}$

Space group, $P 6_{3} m c$; refined unit cell parameters for FeS IV: $2 a=6.7175(10) \AA, c=5.6542(12) \AA$; unit cell parameter for gold, $a=4.0510$ (7) $\AA$, corresponding to a pressure of $5.2 \mathrm{GPa}$. 
Table 1b. Refined parameters for FeS IV phase at $9.0 \mathrm{GPa}$ and $530 \mathrm{~K}$ derived from Rietveld refinement.

\begin{tabular}{lllll}
\hline atom & \multicolumn{1}{c}{$\mathrm{x}$} & $\mathrm{y}$ & $\mathrm{z}$ & $U_{\text {iso }} \times 10^{2}$ \\
& & & & \\
$\mathrm{Fe}(1)$ & 0.5211 & 0.0422 & 0 & 2.2 \\
$\mathrm{Fe}(2)$ & 0 & 0 & -0.0156 & 3.5 \\
$\mathrm{~S}(1)$ & 0.1666 & 0.3332 & 0.2358 & 1.7 \\
$\mathrm{~S}(2)$ & 0.3333 & 0.6667 & 0.7792 & 0.8 \\
\hline
\end{tabular}

Space group, $P 6_{3} m c$; refined unit cell parameters for FeS IV: $2 a=6.5879(10) \AA, c=5.3998(12) \AA$; unit cell parameter for gold, $a=4.0243$ (7) $\AA$, corresponding to a pressure of $9.0 \mathrm{GPa}$.

\section{Hexagonal high-temperature phase, FeS V}

With further increasing temperature, the hexagonal phase FeS IV with a $(2 a, c)$ unit cell transforms to a simple NiAstype hexagonal structure with a $(a, c)$ unit cell (assigned as FeS V) [1, 2]. We collected a diffraction pattern at $3.4 \mathrm{GPa}$ and 650 $\mathrm{K}$ (Fig. 5). The diffraction pattern of $\mathrm{FeS} \mathrm{V}$ is simpler than that of the FeS IV phase. The transition was marked by the extinction of reflections related to the $(2 a, c)$ superlattice, which were indexed as 101, 112, 211, 301, and 103 in the FeS IV phase. The refined lattice parameters for FeS $\mathrm{V}$ are $a=$ $3.4386(8) \AA$ and $c=5.6760(8) \AA$ at $3.4 \mathrm{GPa}$ and $650 \mathrm{~K}$. The Rietveld refinement was based on space group $P 6_{3} / \mathrm{mmc}$. Figure 5 shows the observed and calculated diffraction pattern.

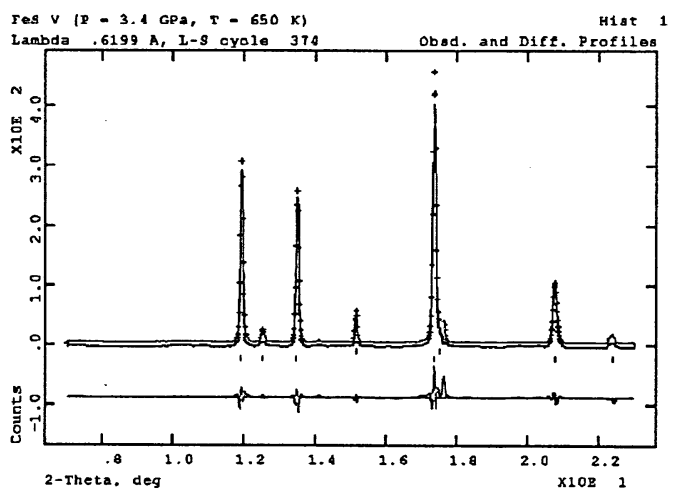

Fig. 5. Observed (crosses) and calculated (solid line) x-ray diffraction pattern for FeS V at $3.4 \mathrm{GPa}$ and $650 \mathrm{~K}$. Tick marks for gold (pressure calibrant) and the $\mathrm{FeS} \mathrm{V}$ phase are shown below the pattern. The difference curve is shown at the bottom.

\section{Conclusion}

High-quality $\mathrm{x}$-ray diffraction data at high pressure and temperature were obtained by combining the image plate and high-temperature diamond-anvil cell techniques. The diffraction patterns were used for structure refinements. The refined unit cell parameters are $a=8.025(4) \AA, b=5.614(2) \AA, c=$ $6.414(4) \AA$, and $\beta=93.01(4)^{\circ}$ at $10.95 \mathrm{GPa}$ and $300 \mathrm{~K}$ for the monoclinic phase, $2 a=6.7175(10) \AA$ and $c=5.6542(12) \AA$ at $5.2 \mathrm{GPa}$ and $530 \mathrm{~K}$ for the hexagonal phase with a superlattice, and $a=3.4386(8) \AA$ and $c=5.6760(8) \AA$ at $3.4 \mathrm{GPa}$ and $650 \mathrm{~K}$ for the simple hexagonal phase. Within the stability field of the $(2 a, c)$ hexagonal phase, an abrupt shortening of the $c$-axis results in about $4 \%$ density change.

Acknowledgements: This work was supported by the National Science Foundation Center for High Pressure Research, the National Aeronautics and Space Administration (NAGW-3942), and the Carnegie Institution of Washington. We wish to acknowledge the excellent support provided by the personnel at CHESS, which is funded by the National Science Foundation.

\section{References}

[1] Y. Fei, C. T. Prewitt, H. K. Mao, and C. M. Bertka, Science 268, 1892 (1995).

[2] Y. Fei and C. T. Prewitt, in Covalent Ceramics III: Science and Technology of Non-Oxides, A. F. Hepp et al., eds, pp. 223-228, Mat. Res. Soc. Symp. Proc. 410, Materials Research Society, Pittsburgh, 1996.

[3] K. Kusaba, Y. Syono, T. Kikegawa, O. Shimomura, J. Phys. Chem. Solids 58, 241 (1997).

[4] Y. Fei, in Mineral Spectroscopy: A Tribute to Roger G. Burns, M. D. Dyar, C. McCammon, and M. W. Schaefer, eds., pp. 243-254, Special Publication No. 5, Geochemical Society, Houston, 1996.

[5] Y. Fei and H. K. Mao, Science 266, 1678 (1994).

[6] Y. Amemiya, J. Synchrotron Rad. 2, 13 (1995).

[7] M. Kunz, K. Leinenweber, J. B. Parise, T. C. Wu, W. A. Bassett, K. Brister, D. J. Weidner, M. T. Vaughan, and Y. Wang, High Pressure Research 14, 311 (1996).

[8] L. A. Taylor and H. K. Mao, Science 170, 850 (1970).

[9] H. E. Jr. King, D. Virgo, and H. K. Mao, Yb. Carnegie Instn, Wash. 77, 830 (1978).

[10] R. O. Pichulo, J. S. Weaver, and T. Takahashi, Meteoritics 11, 351 (1976).

[11] H. K. Mao, G. Zou, and P. M. Bell, Yb. Carnegie Instn, Wash. 80, 267 (1981).

[12] R. J. Nelmes, M. I. McMahon, S. A. Belmonte, D. R. Allan, M. R. Gibbs, and J. B. Parise, This volume (1998).

[13] F. Keller-Besrest and G. Collin, J. Solid State Chem. 84, 194 (1990).

[14] A. C. Larson and R. B. Von Dreele, GSAS manual, Los Alamos Report (1986). 